\section{Effects of halothane anesthesia on the retention of a passive avoidance task in rats*}

\author{
WILLIAM C. PENROD $\div$ and ROBERT BOICE \\ University of Missouri, Columbia, Mo. 65201
}

One-trial passive avoidance training was given to 126 male albino rats in a 3 by 3 by 2 factorial design, with halothane anesthesia (administered 20 sec after training, $30 \mathrm{~min}$ after training, or not administered), retest interval $(1,4$, or 7 days), and preanesthesia (administered 2 days before training or not administered) as main effects. Results were as follows: (1) 24-h retention in nonpreanesthetized Ss replicated the usual gradient of retrograde amnesia; (2) 4and 7-day scores did not exhibit the similarity to Day 1 scores predicted by consolidation theory; $(3)$ the effects of preanesthesia on retention were marked but did not appear to be due to drug dissociation.

Experimentally induced retrograde amnesia (RA) has become a valuable tool in the study of memory storage and retrieval. $R A$ is defined operationally as a performance decrement in a previously learned task for Ss who received some disruptive treatment, as compared to Ss who received no such treatment. Disruptive agents used include electroconvulsive shock (ECS), spreading cortical depression, brain lesion, drugs, anesthesia, and cold water immersion, among others. Of specific interest in this experiment were the effects of halothane (Fluothane) anesthesia.

The most striking characteristic of RA is the temporal gradient of effectiveness, the period of time between training and treatment being critical. One-trial learning tasks have proven useful because the "time of learning" can be measured as a discrete event. However, there is considerable disagreement as to the length of the critical interval. In addition to the understandable differences between different agents (Pearlman, Sharpless, \& Jarvik, 1961), there are even differences reported between experiments using identical agents (Chorover \& Schiller, 1965; Quartermain, Paolino, \& Miller, 1965).

The most widely accepted theory which attempts to explain RA is one of memory consolidation (McGaugh, 1966). According to the theory, long-term retention is achieved through some time-dependent process

* Based on a thesis submitted by the first author to the Graduate School, University of Missouri, in partial fulfillment of requirements for the MA degree, 1970. The authors thank Professors F. A. South, Space Sciences Research Center, and J.H. Mueller. Department of Psychology, for their assistance.

INow in the U.S. Air Force; send reprint requests to $R$. Boice, Department of Psychology, 209 McAlester Hall, University of Missouri. Columbia, Mo. 65201. by which recently processed information is stored permanently. Presumably, interruption of the process at various stages of completion would result in varying degrees of retention loss. Any retention loss attributed to disruption of this consolidation process would have to be permanent, not transient. Attempts by consolidation theorists to confirm the permanence of RA have been moderately successful within the scope of paradigms tested (Luttges \& McGaugh, 1967), but other independent findings remain unexplained. For example, Lewis, Miller, \& Misanin (1968) found that seemingly lost memories of step-down avoidance training could be "returned" to memory be using simple "reminder" shocks before retesting.

Nielson (1968) has offered another explanation of the $\mathrm{RA}$ effect in terms of brain excitability states, with no reference to memory consolidation. There is evidence that, when the brain excitability level is reduced, CRs conditioned at the original level may be lost (Kawakami \& Sawyer, 1964). Brain excitability thresholds, as measured by the intensity of a stimulus required to produce a cortical response, have been shown to be concentrations in the limbic system (Sudak \& Mass, 1964). Finally, Nielson \& Fleming (1968) applied cold, foot shock, and ECS as stressors to rats and found significant alterations in brain amine levels 4 days after treatment.

Nielson's theory is that ECS and other memory disruptors serve to alter brain thresholds after learning. If retest occurs before brain levels return to those present at learning, a dissociation of learning occurs. Thus, the problem is, ostensibly, one of retrieval, not storage. To test the theory, Nielson (1968) applied ECS related to catecholamine before, as well as after, training. Consequently, brain excitability levels should have been elevated during both learning and testing. As predicted, no retention loss was observed.

The purposes of this experiment were to determine if halothane anesthesia acts as a memory disruptor for passive avoidance behavior and to see if the data obtained contradict Nielson's excitability hypothesis. Halothane may be less disruptive than other anesthetics because it induces anesthesia rapidly, minimizing S's struggling, and because it produces fewer irritating side effects to the cardiovascular and respiratory systems (Luschei \& Mehaffey, 1967). In particular, the cerebral blood flow is maintained intact. Of secondary interest in the experiment was the desire to find an anesthetic that does not affect memory while relieving pain. Such an anesthetic would be useful in studying stress (cold water immersion) without the effects of pain.

The Ss were 126 male adult Sprague-Dawley albino rats, weighing between 175 and $250 \mathrm{~g}$. They were housed separately, with free access to lab chow and water.

\section{APPARATUS}

The shuttlebox was divided into $45 \times 45 \times 15 \mathrm{~cm}$ chambers by a guillotine door. One chamber was white and the other, black. Electric shock was provided in the black chamber through stainless steel rods, spaced $1 / 2$ in. apart. Current, at $200 \mathrm{~mA}$, passed through neon bulbs, connected in series with the rods, until an $S$ contacted any two of the rods.

Anesthesia was induced in a closed glass container. Cotton soaked in halothane and covered by a wire screen in the bottom of the container maintained a $5 \%$ concentration of halothane gas at room temperature, due to the vapor pressure of the gas.

PROCEDURE

The experiment was based on a 3 by 3 by 2 factorial design. The first variable was posttrial anesthesia: none, $20-\mathrm{sec}$ delay, or $30-\mathrm{min}$ delay. The second variable was retest interval: 1 , 4 , or 7 days. The third variable was pretrial anesthesia: yes or no. Seven Ss were assigned randomly to each cell.

The Ss were trained in a one-trial passive avoidance situation. Each $\mathrm{S}$ was lowered into the illuminated white side of the shuttlebox, facing away from the door, with a clear plastic handling box (Tighe, 1965). After the $h$ andling box was removed, the guillotine door was opened. The time required for $S$ to cross to the black side was recorded as the initial crossing latency. Ss were shocked upon crossing and escaped back to the white 
side, terminating the trial. Ss were immediately removed from the shuttlebox with the handling box.

For those $S s$ receiving posttrial anesthesia, it was administered either $20 \mathrm{sec}$ or $30 \mathrm{~min}$ after termination of the training trial, depending on the condition. Ss receiving pretrial anesthesia were treated 2 days before the training trial.

Anesthesia was induced by placing individual $S s$ into the closed jar described above. The jar was tilted every few seconds to check for the behavioral signs of anesthesia. Criterion for anesthesia was the ce: sation of all movement after $\mathbf{S}$ lost the righting reflex. After anesthesia, Ss were returned to their cages to recover. Several measures were recorded for both pre- and posttrial anesthetization for Ss receiving those treatments. Those measures were: (1) the time required for $S$ to lose the righting reflex after placement in the anesthesia jar; (2) the time until $S$ stopped all movement; and (3) the time required for $S$ to right itself after removal from the jar.

The Ss were divided into three retest groups, to be tested 1,4 , or 7 days after training, respectively. The retest procedure was identical to training. Retest crossing latencies were measured as in initial crossing. RESULTS

Initial crossing latencies were evenly distributed among the conditions. The basic measure used in this study was the difference between the retest crossing latency and the initial crossing latency. Mean latency scores are plotted in Fig. 1. If $\mathrm{S}$ remained on the white side (avoided) longer on retest, resulting in a positive difference, this was taken as a measure of retention of the learning experience. Significant negative scores were observed as a result of error variances about positive means.

The difference scores gave rise to a large heterogeneity of within-cell variance between cells. Cell variance increased with the magnitude of the cell mean. To permit the use of analysis of variance, a logarithmic (base 10) transformation was employed to provide homogeneous within-cell variance. Each difference score was increased by 20 sec before taking the logarithm to eliminate negative values. The log scores were analyzed in a 3 by 3 by 2 analysis of variance, assuming fixed effects. All three main effects were significant (retest interval, $\mathrm{F}=4.46, \mathrm{df}=2 / 108$, p <. $05 ;$ preanesthesia, $F=4.89$, df $=1 / 108, p<.05$ ), with the posttrial anesthesia being most salient $(F=48.87, \mathrm{df}=2 / 108, \mathrm{p}<.001)$. The two.way interaction between anesthesia and retest interval

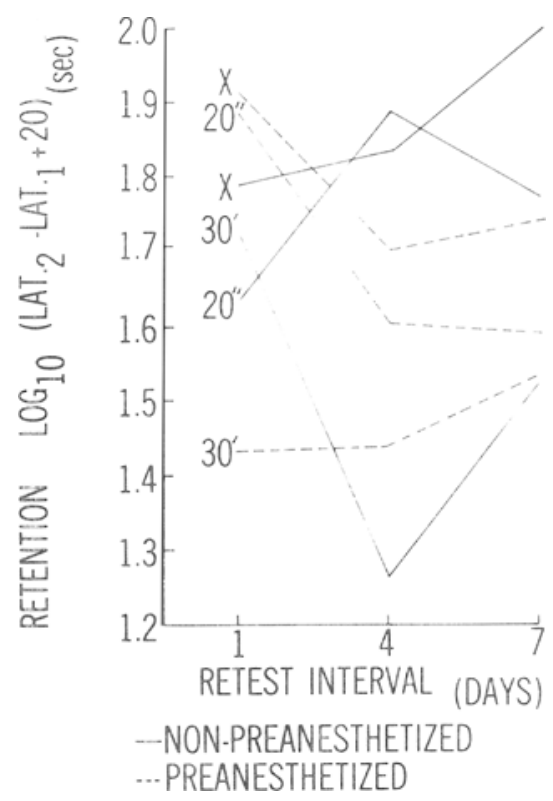

Fig. 1. Transformed crossing latencies. Treatments: $X=$ no posttraining anesthesia; $20^{\prime \prime}=$ anesthesia 20 sec after training; $30^{\prime}$ = anesthesia $30 \mathrm{~min}$ after training.

$(\mathrm{F}=2.63, \mathrm{df}=4 / 108, \mathrm{p}<.05)$ and the three-way interaction $(F=9.57$, $\mathrm{df}=4 / 108, \mathrm{p}<.01)$ were also significant.

The time required for anesthetization (induction) varied slightly between $\mathrm{Ss}$, with the median time being about 20 sec. There was no significant correlation between induction time and latency measures. The recovery time, perhaps a measure of depth of anesthesia, also failed to correlate significantly with retention. Finally, induction time and depth of anesthesia were uncorrelated.

The results lend only partial support to the various theories concerning memory disruption, including the popular consolidation theory. Considering only the nonpreanesthetized conditions, the $24-h$ retest procedure appears to replicate the usual $R A$ gradient found in consolidation studies. Control Ss retained best, followed closely by delayed (30-min) disruption Ss, and lastly, immediate (20-soc) disruption Ss. However, no significant differences were found between the three means, using Duncan's (1955) multiple range test. Four- and 7 -day results are in conflict with conventional consolidation predictions. The most obvious difficulties are the drastic changes in retention of $30-\mathrm{min}$ Ss over the course of 7 days. Again, no statistically reliable differences were found between control and 20-sec groups, but the $30-\mathrm{min}$ Ss were reliably different from both those groups on Days 4 and 7 . The permanence or stability of RA, which is predicted by consolidation theory, is not supported by these data.

Nielson proposed that ECS and other amnesic agents served to alter brain amine levels and brain excitability thresholds, causing a dissociation of learning at recall. In this study, preanesthesia (analogous to pre-ECS) did have a significant effect on retention, but those effects did not agree with Nielson's state-dependency predictions. On the other hand, there is considerable agreement between the obtained results and the data presented by Nielson (1968, p. 15) without explanation. The drastic drop in retention for 30-min nonpreanesthetized $S s$ at 4 days in the present study is paralleled by a drop by Nielson's 4-h non-pre-ECS Ss at 4 days. Nielson also obtained differential results if pre-ECS was administered $1 \mathrm{sec} v \mathrm{~h}$ h after the last familiarization trial, a result totally unexplained before or after the experiment. Nielson's theory may be inapplicable to halothane anesthesia because there is evidence (Cristoforo \& Brody, 1968) that halothane does not act through the release of catecholamines. Since preanesthesia had a marked effect, though not as Nielson would predict, there is reason to doubt if Nielson's interpretation is correct for the ECS situation as well. DISCUSSION

The failure of any single theory to encompass all the data suggests a multiphase memory process. Paolino, Quartermain, \& Levy (1969) varied disruption intensity in an attempt to reconcile apparent differences in $\mathrm{RA}$ gradients between ECS and $\mathrm{CO}_{2}$ inhalation. They found that the differences could not be explained in terms of some common disruption parameter. Instead, it was suggested that different agents such as ECS and $\mathrm{CO}_{2}$ might cause RA by affecting different phases of the consolidation process. Thus, agents could be classified by their physiological effects on the organism such as those listed for halothane above. Any physiological theory such as Nielson's (1968) would have to be agent specific in its predictions. The question of memory disruption will have to be answered by broader experiments which include disruption and test time variables, as in the present study, as well as agent variables, as in Paolino et al (1969).

In summary, halothane anesthesia does appear to have a marked effect upon the retention of a passive avoidance task in this experiment. The results did not provide complete support for any one theory of 
memory, including Nielson's state dependency, but pointed to possible need of a multimechanism approach. However, the results indicate the possibility of using halothane to eliminate pain from cold-immersion studies. Preanesthetized 20-sec Ss tested at $24 \mathrm{~h}$ (the usual period in immersion studies) showed no memory decrement. Thus, halothane may prove to be a useful tool in the study of memory.

\section{REFERENCES}

CHOROVER，S. \& SCHILLER, P. Short-term retrograde amnesia in rats. Journal of Comparative \& Physiological Psychology, 1965, 59, 73-78

CRISTOFORO, M., \& BRODY, M Noradrenergic vasoconstriction produced by halothane and cyclopropane anesthetics. Anesthesiology, 1968, 29 , $79-92$.

DUNCAN, D. Multiple range and multiple F tests. Biometrics, 1955, 1, 1.42.

KAWAKAMI, H., \& SAWYER, C Conditioned induction of paradoxical sleep in the rabbit, Experimental Neurology, 1964, 9, 470-482.

LEWIS, D., MILLER, R., MISANIN, J. Recovery of memory following amnesia. Nature, 1968, 220, 704-705.

LUSCHEI, E., \& MEHAFFEY, J. Small animal anesthesia with halothane. Journal of A pplied Psychology, 1967, 2. 595-597.
LUTTGES, M., \& McGAUGH, J. Permanence of retrograde amnesia produced by electroconvulsive shock. Science, 1967, 156, 408-410.

McGAUGH, J. Time-dependent processes in memory storage. Science, 1966, 153. 1351-1358.

NIELSON, H. C. Evidence that electroconvulsive shock alters memory retrieval rather than memory consolidation. Experimental Neurology, $1968,20,3-20$.

NIELSON, H. C. \& FLEMING, R. Effects of electroconvulsive shock and prior stress levels on brain amine levels. Experimental Neurology, 1968, 20 , 21-30.

PAOLINO, R., QUARTERMAIN, D., \& LEVY, H. Effect of electroconvulsive shock duration on the gradient of retrograde amnesia. Physiology and Behavior, 1969, 4, 147-149.

PEARLMAN, C. A., JR., SHARPLESS, S. \& JARVIK, M. E. Retrograde amnesia produced by anesthetic and convulsant agents. Journal of Comparative \& Physiological Psychology, 1961. 54. 109-112.

QUARTERMAIN, D., PAOLINO, R., \& MILLER, N. A brief temporal gradient of retrograde amnesia independent of situational change, Science, 1965, 149. 116.118.

S U D A K , H . \& M A A S J . Behavioral-neurochemical correlation in reactive and nonreactive strains of rats. Science, $1964,146,418-420$.

TIGHE, T. J. A handling device for small animals. Journal of Experimental Analysis of Behavior, 1965, 8, 261-262.

Amsel, Hug, \& Surridge, 1968; Brooks, 1969) have shown that at least some of the evidence can be accounted for by the frustration hypothesis.

According to the sequential hypothesis (Capaldi, 1967), the aftereffect or stimulus trace occurring on nonrewarded (N) trials becomes conditioned on rewarded (R) trials. Thus, in the sequence NRNR, the animal acquires some habit to run to the $N$ stimulus in that the $N$ aftereffect receives two conditionings. Since from acquisition to extinction there is less generalization decrement of habit strength for partial reward (PR) training than for continuous reward (CR) training, $P R$ is expected to result in greater resistance to extinction.

In order to obtain the PREE in Amsel's system, $N$ trials must first elicit frustration $\left(R_{F}\right)$, which subsequently and in anticipatory form $\left(\mathrm{r}_{\mathrm{F}}-\mathrm{S}_{\mathrm{F}}\right)$ becomes counterconditioned to the approach response $\left(s_{F} \rightarrow R_{A P P}\right)$. Inasmuch as $R_{F}$ requires that anticipatory reward $\left(r_{R}-s_{R}\right)$ be conditioned to the runway cues with some minimum strength, $\mathrm{N}$ trials must be preceded by some number of $R$ trials. If $P R$ training consists only of one NRNR sequence, it follows that the first $N$ trial cannot produce $R_{F}$. Assuming that the second $\mathrm{N}$ trial does produce $R_{F}$, it remains questionable whether sufficient $s_{F} \rightarrow R_{A P P}$ counterconditioning could occur on the one remaining $R$ trial to have an appreciable effect on extinction performance. However, if $\mathrm{CR}$ training were given after the NRNR sequence, it might be expected to enhance counterconditioning of the available $\mathrm{r}_{\mathrm{F}}-\mathrm{S}_{\mathrm{F}}$ and produce the PREE. On the other hand, more $\mathbf{R}$ trials would not be expected to enhance the PREE, given Capaldi's analysis, since the $R$ trial replaces the $N$ aftereffect and removes the occasion for further conditioning of the $\mathrm{N}$ aftereffect.

The present study was conducted to assess the explanatory roles of the frustration and sequential hypotheses in the small-trial PREE by providing interpolated $C R$ training between NRNR or RRRR reward sequences and extinction of an instrumental running response. was observed. The results were interpreted as supporting a counterconditioning but not a sequential model.

The partial reinforcement extinction effect (PREE) following limited training appears readily

\footnotetext{
*Supported by Grant GB-14990X from the National Science Foundation to Abram Amsel. The authors wish to express their appreciation to Mark Kristen and Larry Berry who aided in collecting the data. Requests for reprints should be addressed to Requests for reprints should be addressed to Psychology, University of Texas at Austin, Austin. Tex. 78712 .
}

explainable by Capaldi's sequential hypothesis (e.g., Capaldi, 1967) but poses difficulty for Amsel's frustration hypothesis $(1958,1962,1967)$. Further, Capaldi, McCain, and their associates have amassed a considerable amount of evidence in small-trial situations supporting the sequential interpretation (see Robbins, in press, for a recent review of the small-trial partial reinforcement literature), although some investigators (e.g.,

\section{METHOD}

Ss were 36 male albino rats obtained from Holtzman Farms, Madison, Wisconsin. They were approximately 90 days old at the start of the experiment and were housed individually from the time of their arrival in the laboratory. They were placed on food deprivation approximately 2 weeks before the experiment began. During this period and throughout the experiment, they were given $12 \mathrm{~g}$ of Wayne lab chow 